\title{
A Point Mutation of Adh1 Gene is Involved in the Repression of Coleoptile Elongation under Submergence in Rice
}

\author{
Hiroaki Saika'), Hideo Matsumura $^{2)}$, Tetsuo Takano ${ }^{3)}$, Nobuhiro Tsutsumi ${ }^{1)}$ and Mikio Nakazono*1) \\ 1) Laboratory of Plant Molecular Genetics, Graduate School of Agricultural and Life Sciences, The University of Tokyo, 1-1-1 Yayoi, \\ Bunkyo, Tokyo 113-8657, Japan \\ 2) Iwate Biotechnology Research Center, 22-174-4 Narita, Kitakami, Iwate 024-0003, Japan \\ 3) Asian Natural Environmental Science Center, The University of Tokyo, 1-1-1 Midori, Nishi-Tokyo, Tokyo 188-0002, Japan
}

In higher plants, alcoholic fermentation is required to supply $\mathrm{NAD}^{+}$to the glycolytic pathway, which is responsible for ATP synthesis under anaerobic conditions. Matsumura et al. (1995) previously isolated the reduced adh activity ( $\mathrm{rad})$ mutant in rice, in which elongation of the coleoptile is repressed under submergence. However, the rad gene had not been characterized. In the present study, we observed that, in the coleoptile, the Adh1 mRNA levels were comparable between the rad mutant and the wild type cultivar Kinmaze, while the amount of ADH1 protein was much lower in the rad mutant than in the wild type. Sequencing showed that G106 of the Adh1 gene of Kinmaze was replaced with A in the rad mutant, resulting in an E36K substitution in the deduced amino acid sequence. A genotyping experiment using $\mathrm{F}_{2}$ plants from a cross of the rad mutant with the indica cultivar Kasalath indicated that the point mutation was involved in the repression of coleoptile elongation. Furthermore, since the reduced ADH activity appeared to cause an ATP deficiency, elongation of the coleoptile was repressed in the submerged rad mutant.

Key Words: alcohol dehydrogenase, submergence, rice, coleoptile, ATP deficiency.

\section{Introduction}

Rice (Oryza sativa L.) is one of the few crops that can germinate under complete submergence. The morphology of submerged rice seedlings is markedly different from that of aerobic seedlings. In rice seedlings, that germinate aerobically, the coleoptile emerges from imbibed seeds and elongation stops within a few days. In contrast, leaves and roots continue to grow even after the cessation of coleoptile elongation. On the other hand, in rice seedlings that germinate anaerobically, the elongation of the coleoptile is efficient, unlike that of the leaves and roots. When the elongated coleoptile reaches the water surface, it acts as a snorkel, supplying oxygen to leaves and roots. Therefore, coleoptile elongation is essential for further growth of submerged rice seedlings, including the growth of roots and photosynthetic leaves. In Japan, direct sowing of rice seeds in paddy fields has increasingly replaced the traditional transplanting method because of the reduced costs and labor. Coleoptile growth is necessary for direct sowing to be successful in a submerged field (Ogiwara and Terashima 2001, Miura et al. 2002). Thus, elucidation of the mechanisms of coleoptile elongation might enable to enhance coleoptile growth in rice cultivars that are directly seeded under submerged condi-

Communicated by K. Kadowaki

Received August 18, 2005. Accepted October 19, 2005.

*Corresponding author (e-mail: anakazo@mail.ecc.u-tokyo.ac.jp) tions (Miura et al. 2004).

In many higher plants, alcoholic fermentation is necessary for germination and survival under anaerobic conditions caused by heavy rain and flooding (Tadege et al. 1999). Alcoholic fermentation occurs in two reaction steps (Fig. 1): the decarboxylation of pyruvate to acetaldehyde by pyruvate decarboxylase (PDC) and the subsequent reduction of acetaldehyde to ethanol by alcohol dehydrogenase (ADH). This metabolic pathway supports glycolysis and ATP synthesis by recycling $\mathrm{NAD}^{+}$. Analyses of $\mathrm{ADH}-$ deficient mutants in maize (Schwartz 1969, Freeling and Bennett 1985, Johnson et al. 1994), barley (Harberd and Edwards 1982), Arabidopsis (Jacobs et al. 1988, Ellis et al. 1999) and rice (Matsumura et al. 1998) have shown that $\mathrm{ADH}$ is required for anaerobic tolerance in plants.

When rice germinates under water, ADH activity is required for coleoptile elongation (Setter and Ella 1994, Kato-Noguchi 2001, Kato-Noguchi and Kugimiya 2003). Matsumura et al. (1995) isolated a single recessive rice mutant, whose ADH activity was markedly reduced. Thus, the mutant was designated as reduced adh activity (rad) mutant. Elongation of the coleoptile was strongly repressed in the submerged rad mutant (Matsumura et al. 1995, see Fig. 2A). Subsequently, the amount of ADH1 protein, but not the amount of $\mathrm{ADH} 2$ protein, in shoots and roots in the rad mutant was found to be lower than that in the wild type, while the Adh1 mRNA levels in the rad mutant were comparable to those in the wild type (Matsumura et al. 1998). However, 


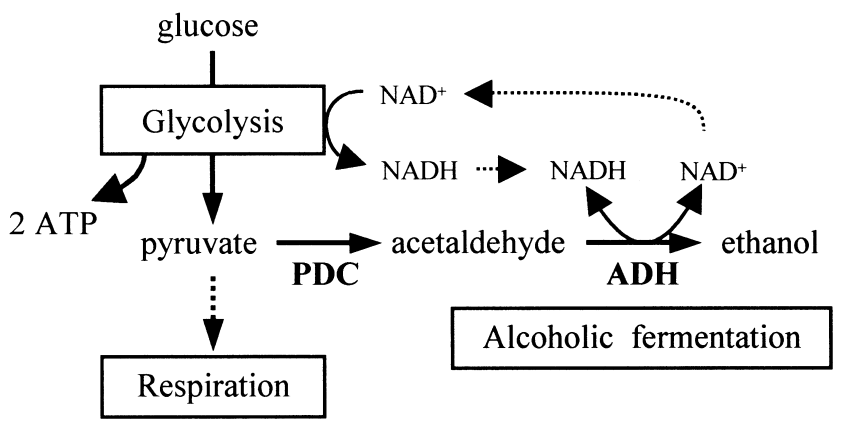

Fig. 1. Diagram of alcoholic fermentation pathway in plants. Alcoholic fermentation occurs in two reaction steps: the decarboxylation of pyruvate to acetaldehyde by PDC and the subsequent reduction of acetaldehyde to ethanol by ADH.

it remains to be determined whether the $A d h 1$ gene showed a similar expression pattern in the rice coleoptile. Furthermore, the gene responsible for the $\mathrm{rad}$ mutation was not identified, although it was assumed to encode ADH1 itself or a protein that regulates ADH1 protein stability (Matsumura et al. 1998).

In the present study, we examined the $A d h 1$ mRNA levels and the ADH protein levels in the coleoptiles in submerged rad mutants. We found that the rad Adhl gene displayed a point mutation linked to the phenotype of repressed coleoptile elongation. Thus, we identified $A d h 1$ as the rad gene. We also found that the reduced ADH activity decreased the ATP content in the coleoptile, suggesting that the reduced ATP amount repressed coleoptile elongation in the submerged rad mutant.

\section{Materials and Methods}

\section{Plant materials, growth conditions and treatments}

Rice (Oryza sativa L., cv. Kinmaze) coleoptiles were grown under complete submergence in darkness at $28^{\circ} \mathrm{C}$. After 5 days, the coleoptiles were harvested, frozen in liquid nitrogen and stored at $-80^{\circ} \mathrm{C}$.

For the genotyping experiment, we used an $\mathrm{F}_{2}$ population consisting of 112 plants derived from the cross indica cultivar 'Kasalath' $\times$ rad mutant. These seeds were germinated under water in darkness at $28^{\circ} \mathrm{C}$ for 4 days. After the length of 4-day-old coleoptiles was measured, the seedlings were transplanted to soil and grown over a one-month period. Total DNA was extracted from 1-month-old leaves and the nucleotide sequences for the $A d h 1$ gene were determined in all the $\mathrm{F}_{2}$ lines.

\section{DNA extraction, PCR and sequence analysis}

Total DNA was extracted from frozen leaves by the benzyl-chloride method (Zhu et al. 1993). The sequence of the $A d h 1$ gene of the rad mutant, from just upstream of the initiation codon to just downstream of the termination codon (referred to as the $A d h 1$ fragment), was first amplified from total DNA by PCR using the Adh1-f1 (f1) and Adh1-r7 (r7) primers (Table 1). The Adh1 fragment was used as the template for direct sequencing with $\mathrm{f} 1$ and $\mathrm{r} 7$ and 12 other forward and reverse primers (Table 1) whose target sequences lay between $\mathrm{f} 1$ and $\mathrm{r} 7$. The sequences were read with an ABI3100 sequencer (Perkin Elmer ABD, Foster City, CA, USA) and were assembled using SEQMAN Software (DNASTAR Inc., Madison, WI, USA).

The Adhl genotype was based on a $496 \mathrm{bp}$ sequence between the $\mathrm{f} 2$ and $\mathrm{r} 2$ target sequences. This sequence was obtained by directly sequencing the Adh1 fragment with the f2 and $\mathrm{r} 2$ primers.
A

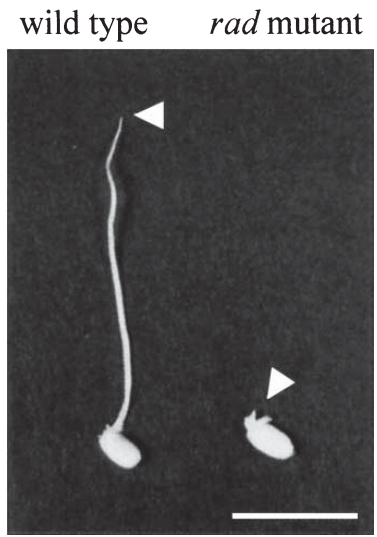

B

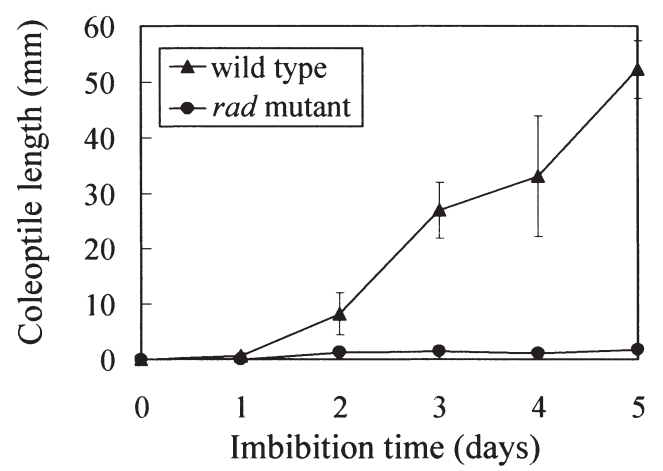

Fig. 2. Comparison of coleoptiles of wild type rice and the rad mutant. (A) Seeds without glumes after imbibition under complete submergence at $28^{\circ} \mathrm{C}$ in darkness for 7 days. Bar $=1 \mathrm{~cm}$. White arrowheads indicate the tips of each coleoptile. (B) Growth of coleoptiles. The length of the coleoptiles of Kinmaze (wild type) and the rad mutant was measured. Values are mean \pm SD for 10 seedlings. 
Table 1. Primers used in the present study

\begin{tabular}{cll}
\hline \hline \multicolumn{2}{c}{ Primer } & \multicolumn{1}{c}{ Sequence (5' to 3') } \\
\hline For sequencing & Adh1-f1 & CTAATAATCCACGCGTGGTG \\
& Adh1-f2 & TGGATTGGGCGAACTTTTAG \\
& Adh1-f3 & CCATGAAGCTGGAGGGTATG \\
& Adh1-f4 & ATGCTGTTGCCTGTGTCAAA \\
& Adh1-f5 & ACAGGGGTGTGATGATTGGT \\
& Adh1-f6 & GACCTGAACGCCAACAGATT \\
& Adh1-f7 & TCTCAAGGGAACCTTCTTCG \\
Adh1-r1 & CTAAAAGTTCGCCCAATCCA \\
& Adh1-r2 & CATACCCTCCAGCTTCATGG \\
Adh1-r3 & TTTGACACAGGCAACAGCAT \\
Adh1-r4 & ACCAATCATCACACCCCTGT \\
Adh1-r5 & AATCTGTTGGCGTTCAGGTC \\
Adh1-r6 & CGAAGAAGGTTCCCTTGAGA \\
Adh1-r7 & AACTCGAGCGCACAAATCTC \\
\hline Adh1-RT-f & TCATCCGCATGGAGAACTA \\
Adh1-RT-r & CCGTATATCATCATTCACCC \\
Pdc1-RT-f & GCAACTGCTGGACAAAGAAG \\
Pdc1-RT-r & AGAAGCTCTTTGCTCGTGTC \\
17SrRNA-RT-f & TCCTACCGATTGAATGGTCC \\
17SrRNA-RT-r & CTTGTTACGACTTCTCCTTCCTC \\
\hline
\end{tabular}

\section{$R N A$ extraction and quantitative $R T-P C R$}

Total RNA was extracted from the frozen coleoptiles using an RNeasy Plant Mini Kit (Qiagen, Valencia, CA, USA). Transcript levels of $A d h 1$ and $P d c 1$ were measured by quantitative RT-PCR using a LightCycler (Roche Diagnostics, Mannheim, Germany) and the QuantiTect SYBR Green RT-PCR kit (Qiagen), according to the manufacturers' protocols. Primers used for RT-PCR are shown in Table 1. A cDNA clone of each gene was used to draw standard curves for quantification. Experiments were repeated twice and the same results were obtained.

\section{Protein extraction and immunoblotting}

Total protein was extracted from 5-day-old coleoptiles by the method of Xie and Wu (1989).

Polyclonal antibodies against rice ADH (Kadowaki et al. 1988) were provided by Dr. K. Kadowaki (National Institute of Agrobiological Sciences, Tsukuba, Japan).

Polyclonal antibodies against rice PDC were prepared as follows. An oligopeptide corresponding to an internal sequence of rice PDC $\left(\mathrm{NH}_{2}-(\mathrm{GC})\right.$ EVIAHKDDTSKELLE$\mathrm{COOH}$ ) was synthesized, purified and injected into rabbits, at Biologica Co. (Nagoya, Japan). The PDC-specific antibodies were purified at Biologica $\mathrm{Co}$. by the following method. The antiserum was loaded onto a synthetic peptidecoupled NHS-activated Sepharose 4 Fast Flow column (Pharmacia Biotech, Uppsala, Sweden), according to the manufacturer's instructions. Antibodies were eluted with $100 \mathrm{mM}$ glycine- $\mathrm{HCl}(\mathrm{pH} 2.5)$ and $150 \mathrm{mM} \mathrm{NaCl}$, and were neutralized with $1 \mathrm{M}$ Tris- $\mathrm{HCl}(\mathrm{pH} \mathrm{8.0)}$. The purified antibodies were stored at $-80^{\circ} \mathrm{C}$.

Total protein was denatured and separated by SDSPAGE. Each lane was loaded with $20 \mu \mathrm{g}$ of protein. Acryl- amide concentrations were $4.75 \%$ and $10 \%(\mathrm{w} / \mathrm{v})$ in the stacking and separation gels, respectively. Immunoblotting was performed with rice ADH-specific antibodies (Kadowaki et al. 1988) and rice PDC-specific antibodies (this study) according to the method of Nakazono et al. (2000). Signal intensities were quantified using LAS-1000 (FUJIFILM, Tokyo, Japan).

\section{Extraction and measurement of ATP}

ATP was extracted according to the method of Saglio and Pradet (1980). Ten frozen coleoptiles were homogenized and mixed with $70 \mu \mathrm{l}$ of $0.6 \mathrm{M}$ trichloroacetic acid (TCA) in diethylether and $700 \mu 1$ of $0.6 \mathrm{M}$ TCA in $\mathrm{H}_{2} \mathrm{O}$. The homogenate was centrifuged at $13,000 \times \mathrm{g}$ for $10 \mathrm{~min}$ at $4{ }^{\circ} \mathrm{C}$. TCA was removed three times from the supernatant with $350 \mu \mathrm{l}$ diethylether. The supernatant was then neutralized with $300 \mu \mathrm{l}$ of $0.5 \mathrm{M}$ Tris-acetate ( $\mathrm{pH} 7.75$ ). ATP content was measured using an ATP assay system LL100-1 (TOYO Bnet, Tokyo, Japan), according to the supplier's instructions.

\section{Results and Discussion}

Expression of Adh1 gene in the coleoptiles of the rad mutant Under submerged conditions, the coleoptiles of the wild type Kinmaze began to elongate one day after imbibition and reached an average length of $52.2 \mathrm{~mm}$ after 5 days (Fig. 2B). However, the coleoptiles of the rad mutant hardly elongated even after 5 days (Fig. 2A and 2B). This result was consistent with the findings of Matsumura et al. (1995).

The relative transcript levels of $A d h 1$ in the coleoptile were comparable between the rad mutant and Kinmaze (Fig. 3A), while the amount of ADH protein in the coleoptiles in the rad mutant was 5-fold lower than that in Kinmaze 
A

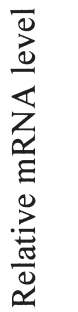

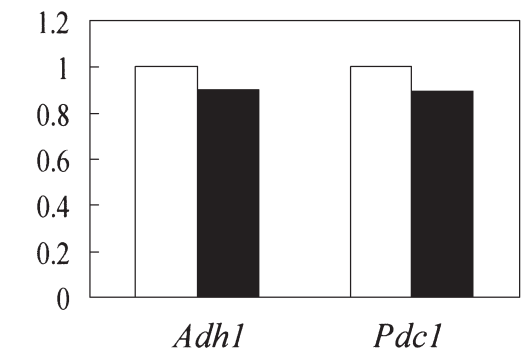

B

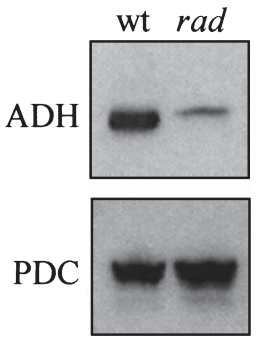

Fig. 3. Gene expression of $A d h$ and $P d c$ in submerged coleoptiles. (A) Quantitative RT-PCR of $A d h 1$ and $P d c 1$ genes. Total RNA was extracted from 5-day-old coleoptiles grown under submergence. For amplification, $0.1 \mu \mathrm{g}$ of total RNA was used as a template. All the mRNA levels were normalized to the 17S rRNA level. (B) Immunoblotting using ADH-specific and PDC-specific antibodies. Total protein was extracted from 5-day-old coleoptiles grown under submergence. For each treatment, total protein $(20 \mu \mathrm{g})$ was used to determine the enzyme protein levels. Equal loading of total protein was confirmed by SDS-PAGE.

A

Adhl

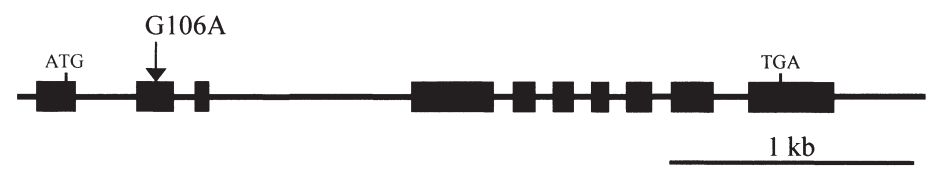

B

Oryza sativa

Zea mays

Arabidopsis thaliana

Caenorhabditis elegans

Drosophila melanogaster

Homo sapiens

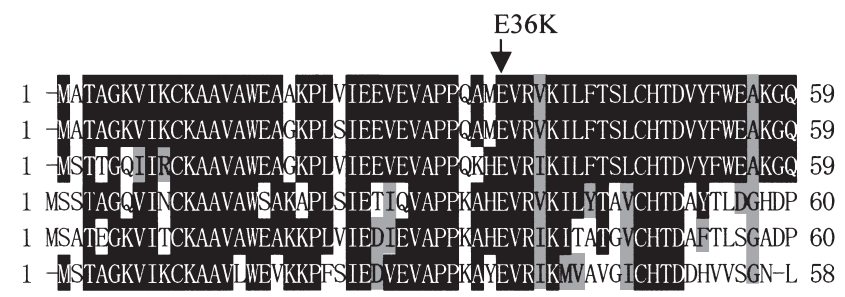

Fig. 4. Analysis of $A d h 1$ gene in the rad mutant. (A) Structure of the $A d h 1$ gene. Black boxes indicate exons. G106 in the wild type was replaced with A in the rad mutant. Bar=1 kb. (B) Alignment of the deduced amino acid sequences of ADH1 proteins from various plant and animal species. Black boxes denote identical amino acids and gray boxes denote similar amino acids. ADH1 proteins were derived Oryza sativa (cultivar Kinmaze, this study), Zea mays (Paul and Ferl 1993, accession no. CAA27681), Arabidopsis thaliana (Innan et al. 1996, accession no. BAA19622), Caenorhabditis elegans (accession no. AAF39899), Drosophila melanogaster (Danielsson et al. 1994, accession no. NM_000668) and Homo sapiens (accession no. AAD37446).

(Fig. 3B). These results were similar to those observed in the leaves and roots of the rad mutant (Matsumura et al. 1998). On the other hand, the $P d c 1$ mRNA levels and the PDC protein levels were comparable between the rad mutant and Kinmaze (Fig. 3A and 3B), suggesting that the expression of the $P d c l$ gene was not affected by the reduction of the ADH protein level.

\section{Characterization of rad gene}

Matsumura et al. $(1995,1998)$ reported that the activation of the ADH1 isozyme was markedly reduced in the rad mutant and suggested that either $A d h 1$ mRNA was not translated or else its product was unstable in the rad plants. A comparison of the sequences of the Adhl genes of the rad mutant and Kinmaze showed that in the rad mutant $\mathrm{G}$ was replaced with $\mathrm{A}$ at the $106^{\text {th }}$ nucleotide from the initiation codon (Fig. 4A), resulting in a predicted replacement of Glu with Lys at position 36 (E36K) (Fig. 4B). We could not find any other mutation in the $A d h 1$ gene in the rad mutant (data not shown). Glu36 in ADH1 is conserved among diverse plant and animal species such as maize (Paul and Ferl 1993), Arabidopsis thaliana (Innan et al. 1996), nematoda, fly (Danielsson et al. 1994) and human (Fig. 4B). This glutamate residue does not appear to be related to the regions that are essential for catalysis [e.g., zinc-binding domains and the pyridine nucleotide-binding domain (Dennis et al. 
A

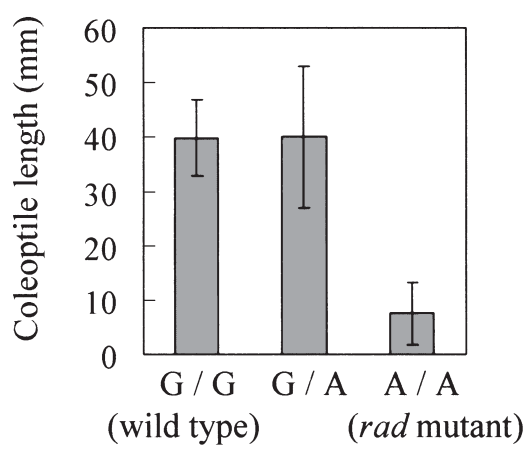

B

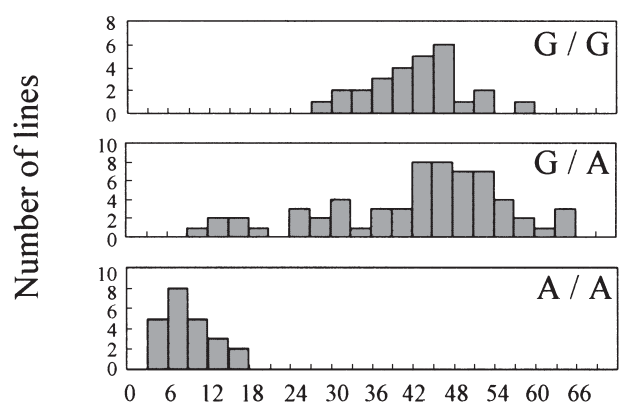

Coleoptile length $(\mathrm{mm})$

Fig. 5. Genotyping of the $A d h 1$ gene. (A) Average length of 4-day-old coleoptiles. Each value is the mean of the length of coleoptiles \pm SD from $27(\mathrm{G} / \mathrm{G}), 62(\mathrm{G} / \mathrm{A})$ and 23 (A/A) seedlings, respectively. (B) Length distribution of 4-day-old coleoptiles of G/G, G/A and A/A seedlings.

1984, Yoshida et al. 2004)]. The structure of the ADH1 proteins of the rad mutant and wild-type, as predicted by the SWISS-MODEL (http://www.expasy.ch/swissmod; Guex and Peitsch 1997) was slightly different (data not shown). If the structure of the ADH1 protein of the rad mutant is different, the change in structure might reduce the protein stability or translational efficiency rather than decrease the enzyme activity. Labeling of the ADH1 protein with a tracer may enable to determine whether the protein stability or translational efficiency is reduced.

Hake et al. (1984) described maize Adhl mutants, in which the ADH1 protein was not detectable, although the Adh1 mRNA level was normal, as in the case of the rad mutant. Since the mutations in these mutants have not been characterized, it remains to be determined whether these mutants have the same defects. Nucleotide sequencing of the $A d h 1$ gene of the maize $A d h 1$ mutant may enable to identify the regions responsible for the stability or translational efficiency of the ADH1 protein.

To confirm that the point mutation $(\mathrm{G} 106 \mathrm{~A})$ of the Adhl gene was correlated with the repressed elongation of the coleoptile in the rad mutant, we genotyped an $\mathrm{F}_{2}$ population consisting of 112 plants generated from a cross of the rad mutant and the indica line 'Kasalath'. The $106^{\text {th }}$ nucleotide from the initiation codon in the $A d h 1$ gene of the rad mutant segregated as G/G (wild type) : G/A : A/A ( rad mu$\operatorname{tant})=27: 62: 23$, which fitted to a $1: 2: 1$ ratio $\left(\chi^{2}=1.57 ; \mathrm{P}\right.$ $>0.05)$. The length of the coleoptile was much shorter in the $\mathrm{A} / \mathrm{A}$ lines than in the $\mathrm{G} / \mathrm{G}$ and $\mathrm{G} / \mathrm{A}$ lines (Fig. 5A and $5 \mathrm{~B}$ ). Therefore, these results indicate that the substitution at position $36(\mathrm{E} 36 \mathrm{~K})$ affected the amount of $\mathrm{ADH} 1$ protein and thus the elongation of the coleoptile in the rad mutant. This further showed that the rad gene was identical with the $A d h 1$ gene.

Rahman et al. (2001) reported that coleoptile elongation was repressed in an $A d h 1$-antisense transgenic rice plant under anoxic conditions. Moreover, coleoptile elongation was found to be strongly correlated with the ADH activity among Japanese cultivars (Kato-Noguchi 2001). The present findings are consistent with these reports. Therefore, our results confirmed that the ADH activity is essential for coleoptile elongation in submerged rice.

\section{ATP content in the coleoptiles of the rad mutant}

Alcoholic fermentation supplies $\mathrm{NAD}^{+}$for glycolysis in order to generate ATP under anaerobic conditions (Tadege et al. 1999). The ATP contents of the coleoptiles in the rad mutant and Kinmaze were $70.1 \pm 11.8 \mathrm{pmol} / \mathrm{coleoptile}$ and $619.8 \pm 327.7 \mathrm{pmol} /$ coleoptile, respectively. Because ADH is required to generate ATP (Fig. 1), this result suggests that since the reduced ADH activity in the rad mutant caused the ATP-deficiency in the coleoptiles, the elongation of submerged coleoptiles was repressed.

\section{Conclusion}

In the rad mutant, a point mutation (G106A) in the Adh1 gene was identified, indicating that Glu36 of the deduced amino acid sequence was replaced with Lys. Our results suggest that this single-base mutation caused a decrease in the amount of ADH protein and a reduction of the ADH activity. As expected, the ATP level in the rad coleoptile was considerably reduced, because alcoholic fermentation is essential for ATP synthesis by glycolysis under anaerobic conditions. Therefore, ATP deficiency may result in the repression of coleoptile elongation in the submerged rad mutant.

\section{Acknowledgements}

The authors express their appreciation to Professor Y. Nagato (The University of Tokyo) for providing the cross 'Kasalath' $\times$ rad mutant and Dr. S. Arimura (The University of Tokyo) for helpful discussions during the course of this study. This work was partly supported by grants-in-aid from the Ministry of Education, Culture, Sports, Science and 
Technology of Japan, and by a grant from the Ministry of Agriculture, Forestry and Fisheries of Japan.

\section{Literature Cited}

Danielsson,O., S.Atrian, T.Luque, L.Hjelmqvist, R.Gonzalez-Duarte and H.Jornvall (1994) Fundamental molecular differences between alcohol dehydrogenase classes. Proc. Natl. Acad. Sci. USA 91: 4980-4984.

Dennis, E.S., W.L.Gerlach, A.J.Pryor, J.L.Bennetzen, A.Inglis, D. Llewellyn, M.M.Sachs, R.J.Ferl and W.J.Peacock (1984) Molecular analysis of the alcohol dehydrogenase $(A d h l)$ gene of maize. Nucleic Acids Res. 12: 3983-4000.

Ellis,M.H., E.S.Dennis and W.J.Peacock (1999) Arabidopsis roots and shoots have different mechanisms for hypoxic stress tolerance. Plant Physiol. 119: 57-64.

Freeling,M. and D.C.Bennett (1985) Maize Adh1. Ann. Rev. Genet. 19: 297-323.

Guex, N. and M.C.Peitsch (1997) SWISS-MODEL and the SwissPdbViewer: an environment for comparative protein modeling. Electrophoresis 18: 2714-2723.

Hake, S., W.C.Taylor and M.Freeling (1984) Molecular analyses of genetically stable mutants of the maize $A d h 1$ gene. Mol. Gen. Genet. 194: 42-48.

Harberd, N.P. and K.J.R.Edwards (1982) The effect of a mutation causing alcohol dehydrogenase deficiency on flooding tolerance in barley. New Phytol. 90: 631-644.

Innan,H., F.Tajima, R.Terauchi and N.T.Miyashita (1996) Intragenic recombination in the Adh locus of the wild plant Arabidopsis thaliana. Genetics 143: 1761-1770.

Jacobs, M., R.Dolferus and D.Van den Bossche (1988) Isolation and biochemical analysis of ethyl methanesulfonate-induced alcohol dehydrogenase null mutants of Arabidopsis thaliana (L.) Heynh. Biochem. Genet. 26: 105-122.

Johnson, J.R., B.G.Cobb and M.C.Drew (1994) Hypoxic induction of anoxia tolerance in roots of Adh1 null Zea mays L. Plant Physiol. 105: 61-67.

Kadowaki,K., M.Matsuoka, N.Murai and K.Harada (1988) Induction of two alcohol dehydrogenase polypeptides in rice roots during anaerobiosis. Plant Sci. 54: 29-36.

Kato-Noguchi,H. (2001) Submergence tolerance and ethanolic fermentation in rice coleoptiles. Plant Prod. Sci. 4: 62-65.

Kato-Noguchi,H. and T.Kugimiya (2003) Preferential induction of alcohol dehydrogenase in coleoptiles of rice seedlings germinated in submergence condition. Biol. Plant. 46: 153-155.

Matsumura,H., T.Takano, K.T.Yoshida and G.Takeda (1995) A rice mutant lacking Alcohol-dehydrogenase. Breed. Sci. 45: 365367.

Matsumura,H., T.Takano, G.Takeda and H.Uchimiya (1998) Adh1 is transcriptionally active but its translational product is reduced in a rad mutant of rice (Oryza sativa L.), which is vulnerable to submergence stress. Theor. Appl. Genet. 97: 1197-1203.

Miura, K., M.Kuroki, H.Shimizu and I.Ando (2002) Introduction of the long-coleoptile trait to improve the establishment of directseeded rice in submerged fields in cool climates. Plant Prod. Sci. 5: 219-223.

Miura, K., S.Y.Lin, H.Araki, T.Nagamine, M.Kuroki, H.Shimizu, I.Ando and M.Yano (2004) Genetical studies on germination of seed and seedling establishment for breeding of improved rice varieties suitable for direct seeding culture. JARQ 38: 1-5.

Nakazono,M., H.Tsuji, Y.Li, D. Saisho, S.Arimura, N.Tsutsumi and A.Hirai (2000) Expression of a gene encoding mitochondrial aldehyde dehydrogenase in rice increases under submerged conditions. Plant Physiol. 124: 587-598.

Ogiwara,H. and K.Terashima (2001) A varietal difference in coleoptile growth is correlated with seedling establishment of direct seeded rice in submerged field under low-temperature conditions. Plant Prod. Sci. 4: 166-172.

Paul,A.L. and R.J.Ferl (1993) Osmium tetroxide footprinting of a scaffold attachment region in the maize $A d h 1$ promoter. Plant Mol. Biol. 22: 1145-1151.

Rahman, M., A.Grover, W.J.Peacock, E.S.Dennis and M.H.Ellis (2001) Effects of manipulation of pyruvate decarboxylase and alcohol dehydrogenase levels on the submergence tolerance of rice. Aust. J. Plant Physiol. 28: 1231-1241.

Saglio,P.H. and A.Pradet (1980) Soluble sugars, respiration, and energy charge during aging of excised maize root tips. Plant Physiol. 66: 516-519.

Schwartz,D. (1969) An example of gene fixation resulting from selective advantage in suboptimal conditions. Am. Nat. 103: 479481.

Setter,T.L. and E.S.Ella (1994) Relationship between coleoptile elongation and alcoholic fermentation in rice exposed to anoxia. I. Importance of treatment conditions and different tissues. Ann. Bot. 74: 265-271.

Tadege,M., I.Dupuis and C.Kuhlemeier (1999) Ethanolic fermentation: new functions for an old pathway. Trends Plant Sci. 4: $320-325$.

Xie,Y. and R.Wu (1989) Rice alcohol dehydrogenase genes: anaerobic induction, organ specific expression and characterization of cDNA clones. Plant Mol. Biol. 13: 53-68.

Yoshida,K., N.T.Miyashita and T.Ishii (2004) Nucleotide polymorphism in the Adhl locus region of the wild rice Oryza rufipogon. Theor. Appl. Genet. 109: 1406-1416.

Zhu,H., F.Qu and L.H.Zhu (1993) Isolation of genomic DNAs from plants, fungi and bacteria using benzyl chloride. Nucleic Acids Res. 21: 5279-5280. 